rev.relac.int.estrateg.segur.10(1):89-109,2015

\title{
LA VIOLENCIA VINCULADA A LA CRIMINALIDAD EN BRASIL Y EL PAPEL DE LAS FUERZAS ARMADAS EN LA BÚSQUEDA DE LA SEGURIDAD PÚBLICA
}

\author{
Carolina Sampó** \\ Valeska Troncoso***
}

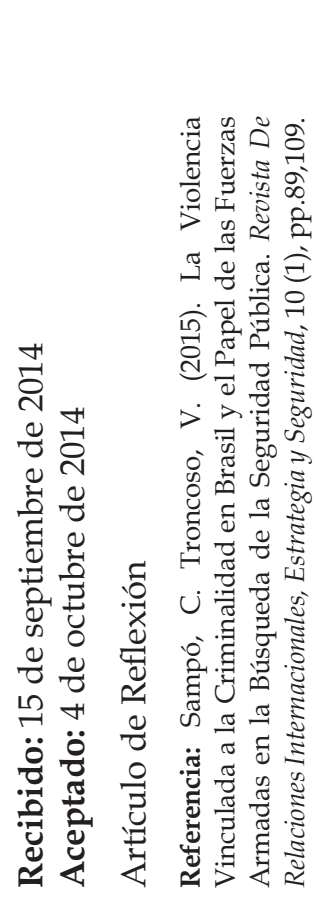

\section{RESUMEN}

La violencia en Brasil es un fenómeno estructural. Sin embargo, el avance de la criminalidad ha sido acompañado por un incremento en los niveles de violencia a punto tal de poner en jaque la integridad territorial forzando al Estado a buscar la manera de mantener el ejercicio de la

\footnotetext{
El presente documento es producto de dos trabajos: uno en curso, la investigación doctoral de Valeska Troncoso titulada "Política de seguridad y defensa en Brasil: el reto de la violencia y la criminalidad en los gobiernos de Luiz Ignacio Lula Da Silva y Dilma Rousseff" y otra, ya finalizada, la investigación posdoctoral de Carolina Sampó titulada "Criminalidad y Violencia: Las Maras centroamericanas y su incidencia en la seguridad regional" financiada por el Consejo Nacional de Investigaciones Científicas y Técnicas (CONICET).

** Doctora en Ciencias Sociales por la Universidad de Buenos Aires (UBA). Magister en Estudios Internacionales (UTDT). Licenciada en Ciencia Política (UBA). Docente de la UBA y de la Universidad de Palermo. Ex becaria doctoral y posdoctoral del Consejo Nacional de Investigaciones Científicas y Técnicas (CONICET). E mail: carosampo@gmail.com

*** Doctoranda en Ciencias Sociales, Universidad de Buenos Aires (UBA). Magíster en Estudios Internacionales, Universidad de Santiago de Chile (USACH) y Licenciada en Historia, Universidad del Desarrollo (UDD). Docente de la Universidad Santo Tomás, Santiago de Chile. E mail: valetroncosoz@gmail.com
} 
soberanía aun en espacios remotos y poco poblados. En los últimos años, la agenda de seguridad brasilera se ha ocupado en gran medida del problema de la violencia asociada a la criminalidad. A pesar de que la problemática se presenta como eminentemente urbana e incluso se concentra en Rio de Janeiro y Sao Paulo, el tamaño y la importancia de esas ciudades han llevado al Poder Ejecutivo a repensar el papel de las Fuerzas Armadas. En este contexto, Brasil ha incorporado tareas consideradas no convencionales a las funciones de las de Fuerzas Armadas como forma de reforzar la Seguridad Pública en tan emblemáticas ciudades. El objetivo del presente trabajo es describir y analizar la situación que atraviesa Brasil en términos de violencia asociada a la criminalidad y el rol que las Fuerzas Armadas han adquirido en ese escenario.

Palabras claves: Violencia, Brasil, Fuerzas Armadas, Seguridad.

\title{
VIOLENCE LINKED TO CRIME IN BRAZIL AND THE ROLE OF THE ARMED FORCES IN THE PURSUIT OF PUBLIC SAFETY
}

\begin{abstract}
Violence in Brazil is a structural phenomenon. However, the advance of criminality has been accompanied by an increase in the levels of violence even jeopardizing the territorial integrity and forcing the State to look for the preservation of sovereignty in remote and inhabit spaces. In the last years, the Brazilian security agenda has been focus in the problem of violence related to criminality. In spite of the fact that the problem is presented as mainly urban and it's concentrated in Rio de Janeiro and Sao Paulo, the size and importance of these cities conducted the Executive Power to rethink the role of Armed Forces. In this scenario, Brazil has incorporated different tasks considered as non-conventional to the functions of the Armed Forces as a way of reinforcing public security in these emblematic cities. The main goal of the present work is to describe and analyze Brasil's situation in terms of violence associated to criminality and the role that the Armed Forces had gained in this scenario.
\end{abstract}

Keywords: Violence, Brazil, Armed Forces, Security.

\section{A VIOLÊNCIA VINCULADA À CRIMINALIDADE NO BRASIL E O PAPEL DAS FORÇAS ARMADAS NA BUSQUEDA DA SEGURANÇA PÚBLICA}

\section{RESUMO}

A violência no Brasil é um fenômeno estrutural. No entanto, o avanço da criminalidade tem sido acompanhada por um aumento nos níveis de violência, ao ponto de pôr em perigo a integridade territorial obrigando ao Estado a procurar manter o exercício da soberania em 
zonas remotas ou de baixa densidade populacional. Nos últimos anos, a agenda de segurança brasileira tem sido maiormente ocupada com o problema da violência associada ao crime. Embora o problema seja apresentado como eminentemente urbano e mesmo concentrado no Rio de Janeiro e no São Paulo, o tamanho e a importância destas cidades levaram ao Executivo a repensar o papel das Forças Armadas. Neste contexto, o Brasil incorporou tarefas não convencionais nas funções das Forças Armadas com o fim de fortalecer a Segurança Pública das cidades emblemáticas. O objetivo deste artigo é descrever e analisar a situação do Brasil em termos de violência associada ao crime e ao papel que as Forças Armadas têm adquirido nesse cenário.

Palavras-chave: Violência, Brasil, Forças Armadas, Segurança.

\section{INTRODUCCIÓN}

Resulta curioso cómo se llevó a cabo el proceso que resultó en el reconocimiento público de la existencia tanto de la violencia como de la criminalidad en Brasil, teniendo en cuenta que se trata de fenómenos que distan de ser nuevos.

Sin embargo, desde el reconocimiento de su existencia, la violencia se ha visto incrementada poderosamente, posicionándose como el problema que genera mayor preocupación social no sólo por su aumento cuantitativo sino también por su progresiva peligrosidad cualitativa (Escalante, 2003). La combinación de altos índices de criminalidad, la violencia interpersonal y la impunidad generalizada, se ven materializados en la sensación de inseguridad que predomina en grandes estratos de la sociedad civil brasilera. En este sentido, la proliferación de drogas ilícitas, excesos a la hora de consumir alcohol y la gran cantidad de armas pequeñas y livianas en circulación, así como las inadecuadas respuestas en el campo de la seguridad pública y el sistema judicial, conformaron el telón de fondo de la violencia (Reichenheim, M. et al, 2011).

Finalmente, es necesario destacar la pobreza extrema que se registra en las favelas especialmente de Rio de Janeiro o Sao Pablo, ya que allí se conforman las fortalezas ideales para el accionar de grupos criminales que organizan el tráfico de cocaína, idean secuestros y deciden sobre la compra de armas aumentando el nivel de exposición del crimen organizado, constituyendo una fuerte amenaza a la gobernabilidad y afectando específicamente a las políticas de seguridad y al sector de la Defensa.

En este contexto, los primeros meses del año 2014 pusieron de manifiesto los problemas, cada vez más profundos, en torno a la violencia que parece ser estructural en la sociedad brasilera. A menos de cinco años del inicio del proyecto pacificador de las favelas cariocas y a pesar del aparente debilitamiento del poder de los narcotraficantes, las principales facciones criminales 
se han atrincherado en zonas periféricas desde las que controlan la venta de drogas en favelas enclavadas estratégicamente (Barón, 2014).

Esta nueva irrupción de la violencia parece deberse al hartazgo de gran parte de la ciudadanía, principalmente habitante de las favelas, que ha sido tratada durante décadas como de segunda categoría pero que se presenta como una mayoría en términos numéricos. Además no debe perderse de vista la consabida atención internacional puesta sobre Brasil por el Mundial de Futbol y los Juegos Olímpicos. Por otro lado, es necesario no perder de vista la reaparición de un clima de tensión en las zonas más turísticas de Rio, propiciado por un nuevo incremento en la delincuencia, la omnipresencia del Comando Vermelho (Vide Infra) y los enfrentamientos de distintos sectores sociales con la Policía.

En este escenario, el presente trabajo se propone describir y analizar la situación que atraviesa Brasil en términos de violencia y el rol que las Fuerzas Armadas (FFAA) adquirieron en los últimos tiempos especialmente teniendo en cuenta los cambios que las involucran más en cuestiones consideradas del ámbito de la Seguridad y no necesariamente de la Defensa. Vale decir que en su Estrategia Nacional de Defensa del año 2008, revisada el año 2012, se sugiere que las amenazas deben ser combatidas y remediadas con recursos militares, cualquiera sea su naturaleza o carácter.

Este artículo se centra en la problemática de la violencia y en la observación de la seguridad desde el punto de vista de la disonancia entre un instrumento militar formal y los problemas del uso de esta fuerza en un escenario de no guerra que está comparativamente menos previsto en la Estrategia antes mencionada. Con este objetivo, este trabajo se dividirá en tres secciones. En la primera se llevará adelante una aproximación al fenómeno de la violencia entendido en términos teóricos. En segundo lugar se analizará el escenario brasilero y, finalmente, se analizará la función de las FFAA en el control de la criminalidad, el delito y la violencia.

\section{UNA APROXIMACIÓN AL FENÓMENO DE LA VIOLENCIA}

La violencia en Brasil, crónica, endémica y estructural, no parece estar asociada al menos de manera directa, al incremento de la criminalidad como sí pasa en el resto de los países latinoamericanos. En realidad, la brecha entre ricos y pobres, consecuencia de la mala distribución de la riqueza es lo que genera una fuerte oposición entre distintos sectores sociales. Las clases más bajas, en su mayoría asentadas en favelas, son las que perpetúan contra otros sectores sociales, acciones violentas. Demostrando de esta forma que, como menciona Cornblit (2001), son situaciones específicas las que favorecen el desarrollo de la violencia siendo que se trata de una condición latente en los seres humanos.

De un tiempo a esta parte, los enfrentamientos entre sectores marginales de la sociedad y la policía han mostrado la relevancia de la problemática para otros segmentos sociales, especialmente 
en ciudades como San Pablo y Rio de Janeiro, que han dejado de ver sólo por televisión la proliferación de la violencia. De allí que es necesario definir a este fenómeno de la forma más acabada posible para entender cómo se desarrolla y qué forma adopta en el país carioca.

En primer lugar, vale decir que la violencia es un elemento constitutivo de una relación social. Puede ser parte de una relación interpersonal, de una familiar o bien puede extenderse más allá hasta involucrar a todos los sectores sociales que conforman la sociedad civil. En el caso de Brasil, la violencia aparece como parte constitutiva de la sociedad pero es la interacción entre los distintos actores que la componen, lo que determina el tipo de violencia frente al que se está y cuál es su alcance. Es decir que tiene una dimensión relacional.

Para el análisis resulta útil la tipología de Galtung (2004), que establece tres clases de violencia: la estructural, la cultural y la directa. Para él, las dos primeras son invisibles, mientras que la directa es visible y es resultado de la violencia cultural y estructural, que aparecen como móviles de la acción humana y emplean actores violentos que se rebelan contra las estructuras y legitiman el uso de la violencia a partir de referencias a la cultura. La violencia directa es visible y queda reflejada en muertos, heridos, daños materiales y desplazados. En cambio, la violencia invisible se alimenta de lo cultural y estructural.

Sin embargo, es necesario destacar que la violencia directa refuerza la violencia estructural y cultural, existentes. De esta manera, la sociedad comienza a reproducir un círculo vicioso que termina por desnudar una única realidad: la violencia sólo puede ser modificada con violencia. De esa forma se genera una nueva estructura violenta que, además, refuerza la cultura del conflicto. De allí que la resolución sea tan compleja, ya que requiere no sólo que el problema que subyace al conflicto sea solucionado completamente, sino que también se lleve adelante una reconstrucción sobre aquello que sufrió violencia directa y se genere una nueva cultura, a partir de la reconciliación de las partes en conflicto (Galtung, 2004).

En el caso de Brasil, aplicando a Galtung, son la violencia estructural y la violencia cultural las que revisten mayor importancia, pues en lo que hace a los indicadores relativos a la violencia directa, el país carioca se encuentra muy cerca de la media latinoamericana. Sin embargo, la cultura de la violencia logra permear a la sociedad reforzando la idea de que su existencia es parte constitutiva de las relaciones sociales.

Concha Eastman (2002, p.504) define la violencia social como: "La expresión de una relación en la que el conflicto que la precede no se resolvió y el o los actores optan por la agresión". En este sentido, como destaca Crettiez (2009), la violencia es intencional y se utiliza como medio para llegar a un fin.

Aunque existen numerosas definiciones de violencia, la mayoría de los autores coinciden en que se trata de una expresión de insatisfacción que se establece como mecanismo de resolución de conflictos, especialmente cuando las instituciones son débiles (Dammert, 2011). Esa violencia, 
visible, es entendida como "la ruptura, por la fuerza desordenada y explosiva, del orden jurídico-social, y que puede, eventualmente, dar lugar a la delincuencia, a la marginalidad o a otras muchas ilegalidades cohibibles por el poder del Estado" (Sodré, 2001, p.18).

Adicionalmente, la violencia social puede expresarse como violencia instrumental o como violencia emocional, según Morrison, Buvinic \& Shifter (2005, p.126) quienes afirman que: "La violencia instrumental es aquella que se usa como medio para obtener un propósito" mientras que la violencia emocional es una respuesta agresiva que no persigue otro fin y no responde a un cálculo racional. En este mismo sentido, Crettiez (2009, p.17) destacó que la violencia puede ser una pulsión que tiene como fin último destruir o ensuciar al otro, sea para satisfacer la ira, el odio o cualquier otro sentimiento negativo. En este último caso, podríamos hablar de violencia pasional ya que "sirve para expresar un furor colectivo o individual, una frustración y una cólera pasajeras".

La violencia puede ser analizada desde, al menos, dos perspectivas: a nivel macro, la violencia subyace a toda relación social. A nivel micro puede entenderse como un medio para alcanzar un fin determinado, en muchos casos como método de resolución de conflictos o bien como producto de una pulsión irracional. De allí que sea necesario aclarar que puede ser entendida como parte constitutiva de la sociedad pero, al mismo tiempo, puede ser un método de resolución de controversias.

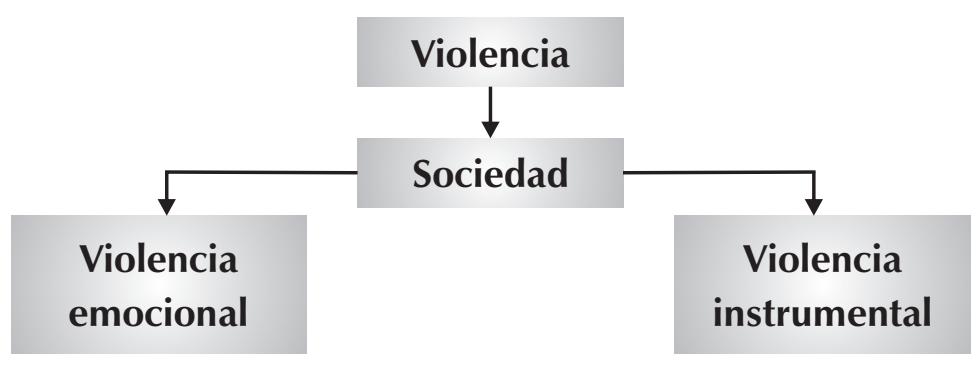

Figura 1. Violencia a niveles micro y macro Fuente: elaboración propia.

En Brasil, la violencia instrumental parece ser la que aparece más frecuentemente. Sin embargo, es necesario no desdeñar la cantidad de casos en los que la violencia emocional aparece, especialmente porque se encuentra relacionada al consumo de alcohol y drogas. En esos momentos, es claro que el comportamiento de los individuos no responde a patrones racionales sino que son variables culturales y psico-sociales las que guían su comportamiento. En este sentido, podemos afirmar que hablamos de la violencia directa de Galtung (2004), que se alimenta de la violencia estructural y de la violencia cultural. De allí que la socialización que reciben estos individuos es clave para comprender los patrones que repiten a través de sus acciones. Las normas y los valores que permiten dirimir lo bueno de lo malo, lo correcto 
de lo incorrecto, no son iguales en todos los sectores sociales y predeterminan el tipo de comportamiento e incluso la forma de vida que desarrolla un individuo.

Pero, ¿por qué los individuos deciden utilizar la violencia? Básicamente por dos motivos: Por un lado porque la carencia de reconocimiento y acceso al poder los impulsa a utilizar la violencia con el objeto de llegar a un Estado que se presenta como lejano y hasta inalcanzable $y$, a partir de allí, exigir un reconocimiento que hasta ese momento era extremadamente limitado. Finalmente, la violencia puede ser la respuesta a una situación económico-social de marginalidad que, al generar cólera y frustración, se presenta como la única alternativa viable.

En este sentido, Crettiez (2009) afirmó que la violencia callejera funciona como un mecanismo de ascenso social para jóvenes que carecen de otras alternativas, que se convierten en interlocutores de poderes y se sienten valorizados a partir de su desempeño, que se convierte en una fuente de reconocimiento social. Este mecanismo inaugura una forma de inclusión estrechamente vinculada al uso de la violencia, que a la vez reproduce las fracturas existentes en la sociedad y reproduce los factores que impiden la resolución de conflictos, perpetuando el uso de la violencia.

\section{LA VIOLENCIA EN BRASIL}

Brasil parece ser un caso testigo de la definición de violencia que plantea Crettiez (2009). La violencia se presenta como un medio para llegar a un fin, al tiempo que se constituye como una forma de ascender socialmente para un sector marginado de jóvenes. El alto número de homicidios da cuenta de los niveles de violencia que penetran a la sociedad brasilera. Según un reciente informe (Fleitas, 2014, pp. 12-14) la tasa de homicidios para el año 2012 fue de alrededor de 25 cada 100 mil habitantes, número que está por encima de la media tanto latinoamericana, que es de 23,5 cada 100 mil, como sudamericana (22,6/100.000 hab.).

Un elemento que permite comprender la escalada de violencia en los centros urbanos brasileros es el incremento del tráfico de drogas. El narcomenudeo, estructurado en la década del ochenta en Rio de Janeiro, tenía como objetivo alcanzar beneficios económicos y mantener la disciplina interna de las organizaciones que lo regenteaban, al tiempo que usaban la violencia como forma de resistencia ante la policía. Así "la violencia relacionada con la droga y con disputas territoriales entre facciones rivales puede ser considerada el principal factor para el aumento en un 140\% de muertes provocadas por armas cortas en la ciudad entre 1979 y 2000" (Mazo \& Lopez, 2009, p.210). En este sentido, es necesario destacar la violencia del crimen organizado como medio para alcanzar sus objetivos.

La importancia del narcotráfico y su penetración en la sociedad brasilera no puede ser desestimada considerando que se trata del segundo país consumidor de cocaína del mundo, detrás de los EEUU (Olinger, 2013), sino también porque comparte límites con los tres principales productores de esta substancia: Perú, Colombia y Bolivia. Además, Paraguay -con quien limita 
al sur- es el principal productor y exportador sudamericano de marihuana y se encarga de nutrir al mercado interno, abastecido por las organizaciones locales que mencionamos antes. De acuerdo al Informe Anual de Drogas de la ONU (2014), Brasil es el país más que presenta más consumo de cocaína en la región latinoamericana.

En este contexto es necesario destacar que Brasil tiene fronteras con nueve de los once países que conforman Sudamérica, hecho que facilita el tráfico de drogas, tanto dentro del subcontinente como fuera de él (siendo África y Europa los principales destinos). Los enormes espacios selváticos y escasamente habitados que se encuentran en el norte y oeste de su territorio, sumados a las vías de transporte fluvial que ofrecen el Pantanal y la cuenca amazónica, contribuyen decisivamente a ese tráfico. En este contexto, la ciudad de Río de Janeiro se ha convertido en uno de los puntos de tránsito más importantes de exportación de cocaína hacia EE.UU., Europa y África del Sur.

Aun cuando la violencia es estructural y crónica en Brasil, es innegable que, por un lado el avance del crimen organizado y, por otro, la reproducción de estructuras locales que facilitan el tráfico y la comercialización de drogas, contribuyeron al incremento de los índices que dan cuenta de ella.

Koonings y Veenstra (2007, p.622) señalan que la expansión de los grupos criminales en Río de Janeiro coincidió con el incremento internacional del comercio de drogas y el aumento del consumo local entre los segmentos medio y alto de la sociedad, consolidándose tres grandes grupos: Comando Vermelho, Amigos dos Amigos y Terceiro Comando. Estas organizaciones en la actualidad controlan el mercado de drogas como la cocaína y la marihuana, actuando en favelas y constituyendo una red con estructura jerárquica en donde se estima que de los aproximadamente 10.000 miembros con los que cuentan, la mitad son menores de edad (Barchechat, 2006, p.6).

La venta de droga en los grandes centros urbanos opera en grupos armados organizados jerárquicamente de forma piramidal que someten a las comunidades de la ciudad a través del dominio territorial y paramilitar.

Como explica Luke Dowdney (2003, p. 39) "las bandas de traficantes de drogas no están estructuradas como se entiende tradicionalmente en un cartel o mafia con un solo personaje líder que toma la única decisión sobre las actividades de un grupo, sino que se les considera como una red de actores independientes afiliados (donos) que se apoyan entre sí con propósitos defensivos u ofensivos". En un lenguaje corriente, Mazo y López (2009, p. 211) señalan que "cada comando o facción tiene representación comercial en varias favelas y se organiza en sucursales y puestos de venta" y cada sucursal está dirigida por un dono.

Pese a su independencia, las bandas de traficantes o donos actúan dentro de una estructura que opera gracias a la acción de diversos actores sin los cuales los donos no existirían, ya que cada uno de ellos cumple un rol determinado. La importación de cocaína, producto de 
los contactos con bandas criminales transnacionales, recae en los llamados atacadistas y el trasporte de cocaína a las favelas controladas por los donos, en los matutos, quienes además proveen a éstos de armas de fuego. Esto significa que los donos dependen completamente de ellos para continuar con su negocio de venta de drogas en las favelas.

Es importante resaltar que las bandas de traficantes de drogas en las principales ciudades de Brasil actúan según un sistema de lealtades entre donos, que están unidos en una red flexible de apoyo mutuo, tanto dentro como fuera del sistema carcelario. En el caso del Comando Vermelho sus donos más poderosos están en prisión, sin embargo, desde ahí controlan sus territorios a través del uso de teléfonos celulares y de las visitas de sus correligionarios. Para Dowdney (2003, p.45):

ya que la mayoría de estos donos se encuentran en la misma prisión de máxima seguridad, Bangu I, al oeste de Río de Janeiro, han podido centralizar el control de las bandas a través de una estructura basada en las prisiones, liderada por un «presidente» y un «vicepresidente». Ellos no controlan o reciben dinero de los territorios de otros donos, en cambio, regulan la vida en la prisión, calman las disputas internas de la banda que tiene lugar fuera de la prisión y toman la decisión final sobre cualquier problema de mutuo interés de los afiliados a la banda.

En este contexto, es importante destacar que, a menudo, actores armados irregulares desempeñan un papel particular en la organización y el funcionamiento de sistemas alternativos e informales de seguridad y orden público; en este sentido, las bandas criminales ligadas al narcotráfico frente a los vacíos de gobernanza formal generalmente instalan alguna forma de orden y control motivados por el temor al aumento de las probabilidades que la policía incremente operativos en la zona y que los habitantes proporcionen información a la policía sobre los ilícitos que se cometen en sus barrios (Koonings \& Veenstra, 2007).

Por ello no es de extrañar que las bandas criminales muestren cierto interés de tipo social por el barrio, proveyendo algunos bienes y servicios comunitarios, configurándose así una red de control de orden público que implica un sometimiento por parte de la población bajo una lógica de violencia. De allí la necesidad de reforzar el ejercicio de la soberanía en todo el territorio, a través del accionar de las FFAA.

Si bien estos grupos no se caracterizan por utilizar la violencia como forma de actuar y abrirse camino, es necesario destacar que sí existe violencia vinculada al consumo de drogas, que se ha incrementado fuertemente en los últimos años. Es decir que la criminalidad y la violencia están vinculadas a través del consumo de estupefacientes. Por eso la Presidente Dilma Rousseff propuso un modelo de desarrollo que no sólo implica un compromiso con la sustentabilidad, el crecimiento económico, el control de la inflación y el aumento del empleo, sino que también da solución a demandas de larga data como la falta de presencia estatal en regiones donde reina la violencia armada y el crimen organizado (Rousseff, 2012a). 
Es necesario señalar que la violencia no solamente se genera en las calles de las grandes ciudades sino que también la violencia entre prisioneros encarcelados es cosa de todos los días; como pudimos ver hace poco durante un motín en el que algunos presos decapitaron a otros y también arrojaron a dos individuos, que murieron posteriormente, desde un techo de 15 metros. Es decir que las prisiones no contribuyen a resolver el problema de la seguridad pública en tanto reproducen conductas violentas, incluso es frecuente que líderes de bandas de narcotráfico coordinen acciones delictivas desde el establecimiento penitenciario (Dammert \& Zúñiga, 2007, p.18).

Dentro del sistema penitenciario han surgido bandas criminales como el Primeiro Comando da Capital (PCC) que tiene su origen, a comienzos de los años 90', en la Penitenciaría Taubaté en San Paulo y que en la actualidad mantiene nexos con bandas criminales de otros países latinoamericanos como Bolivia y Paraguay. Para comprender la consolidación del PCC en el estado de San Paulo es necesario tener en cuenta lo que Elías (2002, p.30) denominó la monopolización de las oportunidades de poder disponibles en las cárceles, vinculada a dos aspectos centrales de las relaciones de poder.

En primer lugar, el poder económico con respecto a las oportunidades comerciales informales/ ilegales que proliferan en el universo penitenciario, las cuales no sólo están ligadas al comercio ilícito de drogas, sino que también al hecho que el PCC regula y controla este comercio, así como las oportunidades de acceso a la misma. En segundo lugar, el poder político, que en este caso significa la prerrogativa del PCC (a través de sus miembros) para ejercer un estricto control social sobre la población reclusa. ${ }^{1}$

Caldeira (2011, p.21) postuló en cuanto al proceso de reconfiguración de las relaciones sociales carcelarias como el "resultado de la monopolización del poder por el PCC, produce efectos drásticos en la forma en que las jerarquías sociales se construyen en este espacio, así como las prácticas que componen la dinámica de la figuración social". Por tanto, el monopolio privado de la violencia produce un nuevo equilibrio de poder, donde las diferencias de poder se amplían radicalmente y se concentran en la posición central que el PCC ocupa dentro de las masas de la prisión (Elias \& Scotson, 2000, p.206).

El monopolio de la violencia es utilizado para el beneficio de quien lo controla y en nombre de la población que es sometida. Así, la violencia fue un mecanismo de base para la resolución de toda clase de conflictos y la capacidad de utilizar la fuerza física se afianzó en este espacio social donde no existía una autoridad central que pudiese ofrecer protección a los más débiles y vulnerables (Caldeira, 2011, p.188).

1. Este control social se basa en la obediencia, no necesariamente consensuada, reglas, normas y formas de conducta establecidas por la organización, cuya contraparte es la definición de los delitos, el enjuiciamiento de los responsables y su correspondiente castigo. 
El caso del PCC evidencia la violencia estructural que afecta a Brasil ya que la inexistencia de un centro de gravitación de poder tiene relación con la ausencia de cualquier forma de regulación de los conflictos por parte de la administración penitenciaria, la cual además carece de legitimidad para hacer que los internos cumplan voluntariamente las órdenes, por tanto, la coerción física se vislumbra como la única manera en que la administración penitenciaria puede hacerlos obedecer. ${ }^{2}$

En resumen, la violencia sea directa o indirecta, física o psicológica, actúa como un mecanismo de presión sobre la sociedad civil (y población carcelaria) y es utilizada por los grupos criminales con el fin de establecer un orden paralelo al estatal; de manera tal que retienen el control sobre un determinado territorio garantizando el buen desarrollo de sus negocios. Los ciudadanos, muchas veces desprotegidos por un Estado ausente se sienten tentados y, a la vez, forzados a conformar un nuevo orden en el que se les garantiza el acceso a bienes y servicios básicos. En síntesis, la violencia garantiza el reinado de las organizacines criminales.

\section{EL ROL DE LAS FUERZAS ARMADAS: LA RESPUESTA DEL ESTADO}

Luego del retorno a la democracia y tras la desactivación de las hipótesis de conflicto regionales, las preocupaciones de seguridad pasaron a estar más relacionadas con cuestiones internas, vinculadas al avance de fenómenos transnacionales como el crimen organizado y el narcotráfico. De hecho, el notable incremento de la violencia y de la delincuencia instaló el tema de la seguridad pública en la agenda tanto política como social (Troncoso, 2014).

2003 puede ser identificado como punto de inflexión en lo referente a las políticas tendientes a controlar y reducir tanto la violencia como el delito. La gestión en el ámbito de la seguridad pública durante el gobierno de Luiz Inazio Lula Da Silva se caracterizó por combinar acciones en áreas de lo más diversas como la salud y la educación, entre otras; áreas capaces de prevenir la violencia, reprimir la criminalidad y restablecer la seguridad en las ciudades de Brasil (Da Silva, 2003).

Con este fin, en 2005 comenzó a implementarse el Sistema Único de Segurança Pública (SUSP) con el objetivo de consolidar la democratización del sistema político y la implantación de una seguridad ciudadana. En resumen, se puso en marcha un conjunto de políticas públicas

2. Este problema no solo se refiere a la ausencia del Estado en las prisiones brasileñas, sino que también, es la forma en que el Estado se desenvuelve dentro de este sistema, que en última instancia, socava su credibilidad y legitimidad para actuar como autoridad central, por encima de las partes, capaz de regular y mediar en los conflictos en el mismo. Su acción distorsionada, ya sea a través de la corrupción de los funcionarios y empleados, ya sea por la brutalidad y la violencia, deslegitima su autoridad moral sobre la población carcelaria, que afecta directamente el orden social de las prisiones (Caldeira, 2011, p.191). 
destinadas a promover la integración y la modernización del aparato de seguridad en todo el territorio, buscando la mayor integración y articulación entre las policías. ${ }^{3}$

A pesar de esta iniciativa, en Rio de Janeiro se hizo necesario implementar políticas alternativas que permitieran solucionar el problema debido a que los índices de violencia no paraban de crecer. En consecuencia, cinco años más tarde se decidió implementar en algunas favelas de la ciudad Unidades de Policía Pacificadora, con el objetivo de garantizar la seguridad local y fundamentalmente terminar con la violencia y la criminalidad ligadas al tráfico de drogas ilícitas. En este sentido, no debe perderse de vista que el Mundial de Futbol y los Juegos Olímpicos destinados a desarrollarse en la ciudad carioca, incrementan la presión para reducir tanto la violencia como la criminalidad. Es importante señalar que la intervención policial articulada por la Secretaría de Seguridad Pública está vinculada al modelo de policía comunitaria y de contención de la violencia más que de erradicación del narcotráfico.

En este contexto, un tema central es el rol que han adquirido las FFAA a partir de los cambios generados en las políticas de seguridad. Históricamente, en lo que hace al ámbito interno, las FFAA han desempeñado actividades tendientes a reducir problemas de orden social, especialmente en las áreas más remotas del país (Ministerio de Defensa de Brasil, 2005). Según consta en el Mensaje de la Presidenta al Congreso Nacional el año 2011, las FFAA deben brindar apoyo a la ciudadanía en situaciones especiales, a saber: a) Apoyo y asistencia a la población en caso de catástrofe; b) Participación en campañas de vacunación o combate de epidemias; c) Reconstrucción de infraestructura urbana y transporte; d) Atención médica en comunidades localizadas en áreas de muy difícil acceso; e) Colaboración con gobiernos estaduales para la mantención de la ley y el orden (Rousseff, 2012b).

En este contexto es necesario precisar que las Hipótesis de Empleo (en adelante HE), señala las circunstancias en las que podrían ser utilizadas las FFAA, contenidas en la Estrategia de 2008, las cuales mencionan sólo tangencialmente las misiones de resguardo de la estabilidad y el orden interno. Para entender este aspecto hay que tener en cuenta que las HE presuponen un peligro muy lejano. Tanto que ya en 1996, con motivo de su primera difusión de política de Defensa, Brasil reconoció que organizaba las FFAA "en torno a capacidades y no sobre enemigos específicos" (Martinic, 2009, p.48).

En este sentido, es necesario destacar la posición oficial: "Los ambientes apuntados en la Estrategia Nacional de Defensa no permiten vislumbrar amenazas militares concretas y definidas, representadas por fuerzas antagónicas de países potencialmente enemigos o de otros agentes no estatales" es por eso que, debido a la incertidumbre global "la preparación de las Fuerzas Armadas debe ser

3. De acuerdo al artículo № 144 de la Constitución Brasileña, las instituciones a cargo de la seguridad pública son: Policía Federal, Policía Rodoviaria Federal, Policía civil, Policía Militar y cuerpos de Bomberos Militares. 
orientada para actuar en el cumplimiento de variadas misiones, en diferentes áreas y escenarios, para respaldar la acción política del Estado" (República Federativa de Brasil, 2008, p.47).

El Libro Blanco de Defensa Nacional (2012) reafirmó esta postura al plantear que, a pesar de los cambios cada vez más acelerados de las últimas décadas, el orden internacional se sigue determinando, predominantemente, por la relación entre los Estados y por tanto, la defensa de Brasil ante potenciales amenazas externas sigue siendo la misión esencial de las FFAA (República Federativa de Brasil, 2012, p.31). Pese a esto, hay un reconocimiento que nuevos temas o retos pasaron a influir en el ambiente internacional y las implicaciones para la protección de la soberanía, relacionadas al problema de las drogas y los delitos conexos, la protección de la biodiversidad, la biopiratería, la defensa cibernética, las tensiones resultantes de la creciente escasez de recursos, los desastres naturales, los ilícitos trasnacionales, los actos terroristas y la actuación de grupos armados al margen de la ley dejan explícita la creciente transversalidad de los temas de seguridad y de defensa (República Federativa de Brasil, 2012, p.32).

En resumen, el escenario internacional y regional emergente luego del fin de la Guerra Fría, así como el crecimiento incesante de la criminalidad obligó a la clase política brasilera a repensar el rol de sus FFAA, reorientándolas y preparándolas ya no sólo para tareas de Defensa Nacional sino también para misiones que involucran el mantenimiento o restablecimiento del orden social, aunque en forma subsidiaria.

Las preocupaciones centrales en torno a la Defensa Nacional pueden verse reflejadas en las HE. En un primer momento puede verse como las HE adoptan un carácter tradicional, enfocado al dominio territorial y marítimo, que muta junto con el avance de la criminalidad en Brasil que obliga a incorporar nuevos escenarios y tareas para las FFAA. En su Estrategia de Defensa Nacional se mencionan tres HE: la Amazonía, las aguas jurisdiccionales y el territorio" (República Federativa de Brasil, 2008, pp.6 y ss). Pero algo más adelante las tareas se multiplican de acuerdo al documento oficial. A partir de allí se incorporan el monitoreo y control del espacio, del territorio y de las aguas brasileñas en circunstancia de paz. Mientras que en otro lugar se agrega la hipótesis de "la defensa de los recursos hídricos" (República Federativa de Brasil, 2008, pp.13-14).

En consecuencia, una observación documental arroja que hay nueve HE, de las cuáles sólo una se refiere al tema de estabilidad institucional -y de forma subsidiaria-, aunque las territoriales y marítimas clásicas de algún modo vuelven al observador sensible al hecho que las FFAA son requeridas para ejercer soberanía efectiva, en especial en áreas donde la presencia del Estado es débil. De cualquier manera, el eje de sus preocupaciones sigue siendo ejercer soberanía ante otros Estados y ante sí mismo. De allí que las FFAA deban trabajar en la recuperación del territorio soberano, hoy en manos del crimen organizado, combatiendo la violencia directa y psicológica que les permite reinar.

Uno de los aportes centrales del presente enfoque documental es resaltar que en las misiones de las FFAA brasileñas, ellas no tienen que ver primariamente con el mantenimiento del orden y 
de la estabilidad institucional del país. Sin embargo, incluso en Brasil la creciente importancia que han tomado estas tareas supletorias respecto de la misión tradicional -específicamente defender el territorio de todo enemigo externo- tiene por campo una suerte de espacio intermedio de acción: las acciones de No Guerra. El apoyo a la seguridad pública, su participación en misiones de paz, y la necesidad de ejercer una estrategia de la presencia, son indicativas del interés estatal de visualizar una serie de misiones que no son las de confrontar Estados tales y cuáles.

De modo que en la EDN del año 2008, se ve reflejado el temor a que el Estado no ejerza el completo dominio sobre el territorio brasilero; problema capaz de inhibir el ascenso del país a las ligas mayores del poder global. Las hipótesis tradicionales -extendidas ahora al territorio marítimo- son objetivos del Poder Ejecutivo pero, dada la lejanía de la posibilidad de enfrentamientos interestatales, la sociedad civil exhibe demandas de seguridad ciudadana mucho más urgentes desde su perspectiva (Exército brasilero, 2010). Dicho de otra manera, las HE de Brasil que han pensado desde el sector de la Defensa no reflejan la participación real de las FFAA en tareas policiales, estableciendo un hiato entre lo que dice el texto y la realidad.

Esta función subsidiaria de las FFAA es parte del nuevo modelo de desarrollo de Brasil, que establece que su contribución es preservar un adecuado ambiente de seguridad para los nuevos desafíos de la nación sean superados, con el fin de garantizar no solamente la soberanía nacional y la integridad territorial, sino que también la proyección hemisférica y global del Estado brasileño (Rousseff, 2012b). En este sentido, las FFAA deben compartir plenamente los valores de justicia, democracia, paz e igualdad, subyacentes en los objetivos internos y externos de Brasil (Rousseff, 2011).

Uno de estos objetivos es combatir incansablemente al crimen organizado, hecho que demuestra la importancia que han adquirido los problemas de seguridad interna y la incapacidad del Estado de resolver los problemas de violencia y criminalidad. Sin duda, contener la violencia e incluso reducirla, es uno de los objetivos de las FFAA en tanto búsqueda de la paz y conservación de un adecuado ambiente de seguridad.

La publicación del Libro Blanco de Defensa Nacional del año 2012 buscó aportar trasparencia -interna y externa- a los criterios de utilización de las FFAAA brasileñas. En este sentido hay un reconocimiento que las operaciones para garantizar la ley y el orden demandan una preparación y un entrenamiento especial, puesto que el uso de las FFAA en este tipo de operaciones es "fundamentalmente diferente, en principio y doctrina, del tradicional uso en misiones relacionadas a la defensa exterior, en las que el foco es actuar sobre fuerzas enemigas, perfectamente identificables en el terreno, normalmente caracterizadas como una fuerza militar armada y uniformizada"(República Federativa de Brasil, 2012, p.159).

Las peculiaridades de este tipo de acción llevaron a la creación de una escuela destinada a estudiar e investigar el tema, que se materializó en el Centro de Instrucción de Operaciones 
de Garantía de la Ley y el Orden (CIOpGLO) y a la promulgación, por parte del Ministerio de Defensa, del documento Garantía de Ley y Orden con la finalidad de establecer orientaciones para el planeamiento y empleo de las FFAA en operaciones de garantía de la Ley y el Orden (OpGLO).

En este último documento se explicita que las OpGLO se caracterizan por ser operaciones de no guerra, puesto que aunque hay empleo del poder militar internamente, no implican un combate real pero puede, en circunstancias especiales, implicar el uso de la fuerza en una manera limitada (Ministerio de Defensa, 2014, p.17).

El empleo de las FFAA en este tipo de operaciones está circunscrito a las siguientes acciones: garantizar el funcionamiento de los servicios esenciales bajo la responsabilidad del cuerpo paralizado; control de las rutas de tráfico; desocupación de las instalaciones o para proteger la infraestructura crítica, asegurando su funcionamiento; garantizar la seguridad de las autoridades y el ferrocarril; garantizar el derecho de ir y venir de la población; impedir la ocupación de las instalaciones de servicios esenciales; prevenir la obstrucción de las vías vitales para el movimiento de personas y mercancías; proteger lugares de votación; proporcionar apoyo logístico a las diferentes agencias; llevar a cabo el registro y la incautación de armas, explosivos, entre otros; llevar a cabo la vigilancia, estableciendo patrullas a pie y motorizado (Ministerio de Defensa, 2014, p.29).

Como puede observarse, estas operaciones implican el uso de las FFAA en diversos tipos de situaciones y actividades, de cara a las distintas formas en que las perturbaciones del orden y las amenazas a la seguridad de las personas y los bienes pueden estar presentes y deben realizarse bajo tres principios: razonabilidad, es decir, compatibilidad entre los medios y fines de la medida; proporcionalidad entre la acción y la reacción del oponente, a fin de no ser excesivo; y legalidad, referida a la necesidad de que las acciones se lleven a cabo de acuerdo con los mandamientos de la ley (Ministerio de Defensa, 2014, p.25).

Es necesario señalar que el uso de las FFAA para garantizar la ley y el orden es de responsabilidad del Presidente de la República, en los términos del artículo 15 de la Ley Complementaria № 97/199947, donde se especifica que después del mensaje del Presidente se activarán los organismos operativos de las FFAA, que desarrollarán de forma episódica, en un área previamente establecida y por tiempo limitado, las acciones de carácter preventivo y represivo necesarias para garantizar el resultado deseado. La legislación destaca el carácter subsidiario de la intervención de las FFAA, que debe ocurrir sólo "después de agotados los instrumentos destinados a la preservación del orden público y de la incolumidad de las personas y del patrimonio", relacionados en el artículo 144 de la Constitución Federal (República Federativa de Brasil, 2012, p.160).

En otras palabras, lo que las FFAA pretenden es enfocarse principalmente en cuestiones de defensa y su participación en operaciones para garantizar la ley y el orden deben desarrollarse en articulación con organismos de seguridad pública federales, de los estados y municipalidades. 
Un ejemplo de la participación de las FFAA brasileñas en operaciones para garantizar la ley y el orden es la llamada Operación Ágata. Esta operación conducida en la franja de frontera en alianza con los Ministerios de Justicia y de Hacienda, es una actividad conjunta de las FFAA brasileñas destinada a combatir delitos transfronterizos y ambientales, en coordinación con otros organismos federales y de los estados. La operación fue elaborada dentro de la concepción del Plan Estratégico de Fronteras, creado por el Decreto № 7.496 del 8 de junio de 2011, cuyos principales objetivos apuntaban a la neutralización del crimen organizado, la reducción de los índices de criminalidad, la cooperación con los países fronterizos y el apoyo a la población en la franja de frontera (República Federativa de Brasil, 2012, p.67).

La participación de efectivos militares en la operación Ágata 1 del año 2011 fue de 3.044, cifra que aumentó a 8.494 el año 2012 con la Operación Ágata 4 (República Federativa de Brasil, 2012, p.67). Lo anterior evidencia un aumento significativo de la participación de las FFAA en operaciones para garantizar la ley y el orden producto en gran parte a la necesidad de brindar una mejor distribución espacial a las organizaciones militares del Ejército por todo el territorio brasileño y aumentar la prevención y represión de los ilícitos trasnacionales en la región amazónica.

Otro ejemplo de uso de las FFAA, ahora en ámbito urbano, es la participación en la Fuerza de Pacificación establecida en un área de comunidades carentes de la periferia de la ciudad de Rio de Janeiro, conocida como complejos de Alemao y de la Penha. En esta misión han participado refuerzos militares y de la Marina, apoyando la entrada en localidades de difícil acceso. A su vez, la Aeronáutica ha prestado apoyo en algunas operaciones.

Además, efectivos del Ejército y fusileros navales participan directamente en la toma del territorio ocupado por las organizaciones criminales (Rousseff, 2012b). Esta política se enmarca en una acción del gobierno por ampliar el periodo de ocupación de las FFAA en los asentamientos de Río de Janeiro, con miras a evitar que se generara una escalada de violencia durante el Mundial de Fútbol y los próximos Juegos Olímpicos.

La Presidente Rousseff ha señalado en reiteradas ocasiones la importancia de las fuerzas de pacificación en las favelas de la ciudad de Río de Janeiro ya que no sólo implican la participación de la policía y las FFAA, sino que también el uso de la tecnología al servicio de la protección de la población y por consiguiente, "el retorno del respeto a las necesidades de la comunidad" (Rousseff, 2013). En esta línea, Rousseff no ha dudado en señalar que la ciudad de Río de Janeiro es vista por todo Brasil como uno de los estados que mejor ha manejado el tema de la violencia debido a los logros de las Unidades de Pacificación (Rousseff, 2013).

4. De las ocho operaciones realizadas entre los años 2011 y 2014, sólo se dispone información oficial de cuatro de ellas correspondientes a los años 2011 y 2012. 


\section{CONCLUSIÓN}

La violencia en Brasil no es un fenómeno nuevo sino que es la expresión de una relación social constitutiva. En este sentido, la dispar distribución del ingreso, que se traduce en una enorme brecha entre ricos y pobres así como también en un gran número de ciudadanos marginales o de segunda, ha contribuido a enraizar a la violencia como mecanismo cultural y legítimo de resolución de conflictos.

Tal como hemos visto, son situaciones específicas como los contrastes sociales que se observan en Rio de Janeiro, donde la favela más grande de Brasil, la Rosinha, se encuentra emplazada morro arriba de uno de los barrios más exclusivos de la ciudad carioca, las que fortalecen la noción del uso de la violencia instrumental. Desafortunadamente, tal como hemos señalado a lo largo del texto, parece imposible erradicar este tipo de violencia sin la utilización de más violencia, en el corto plazo y sin la solución de problemas de fondo que terminan por eclosionar de esta forma.

En Brasil, así como en otros países con instituciones débiles, la violencia aparece como un medio para alcanzar un fin, rompiendo con el orden jurídico y social allí donde existe e implementando un orden paraestatal donde las instituciones no se hacen presentes. De esta manera, se constituyen espacios, en general favelas, donde son las organizaciones criminales las que se encargan de proveer los bienes primarios poniendo en jaque tanto la soberanía como la integridad territorial del Estado.

De allí la importancia de las FFAA a la hora de recuperar el control del territorio. En algunos casos, estas organizaciones utilizan la violencia para recibir reconocimiento del Estado, mientras los jóvenes que se relacionan con ellas buscan canalizar la frustración que les genera la situación de marginalidad en la que se encuentran junto con las casi inexistentes posibilidades de movilidad social. Volverse visibles, es el objetivo en una sociedad que los excluye y la violencia es el medio para lograrlo.

Hacer cumplir la soberanía del Estado y lograr que se mantenga su integridad territorial, es una de las funciones centrales de las FFAA. Sin embargo, también cumplen con misiones subsidiarias que contribuyen al mantenimiento de la estabilidad y el orden. En este sentido, vale destacar que las preocupaciones vinculadas a la violencia y el delito se instalaron en la agenda social y política debido a su incansable incremento, impulsando el uso de las FFAA en áreas muy disimiles como la salud y la educación.

La Seguridad Pública se ha convertido en una preocupación central tanto para la sociedad como para el gobierno. Por eso las FFAA han sido reorientadas y entrenadas para cumplir funciones de apoyo en tareas policiales tendientes a combatir la violencia y el delito. Esas funciones apuntan a reducir problemas sociales brindando apoyo y colaboración en tareas como la reconstrucción, la distribución de ayuda en caso de catástrofes, la atención médica y el mantenimiento de la ley y el orden. 
Brasil ha dejado claras sus Hipótesis de Empleo y ha incorporado en el Libro Blanco de Defensa Nacional los criterios de utilización de las FFAA, proveyendo un marco de transparencia y dejando en claro que el uso de las FFAA en lo referente al mantenimiento de la estabilidad y el orden es el último recurso, potestad del Presidente de la Nación.

Se ha establecido un modelo de desarrollo que busca preservar un ambiente de seguridad adecuado para garantizar tanto la integridad como la soberanía territorial. Ahora, si los poderes constituidos no consiguen garantizar la paz pública, el Poder Ejecutivo está en capacidad de repensar el rol de las FFAA en operaciones internas que busquen garantizar la ley y el orden. En consecuencia, las FFAA deben estar preparadas para los desafíos contemporáneos, cultivando algunos predicados atribuidos a fuerzas no convencionales.

\section{REFERENCIAS}

Barchechat, O. (2006). Les gangs de rue: premiers éléments d'un état des lieux international. Montreal: Centre international pour la prévention de la criminalité.

Barón, F. (2014, abril 23). Brasil entra en una crisis de seguridad. Diario El País. Recuperado de: http://internacional.elpais.com/internacional/2014/04/23/actualidad/1398282283_ 805055.html entrado 17/07/2014 Consultado el 26.8.2014.

Caldeira, C. (2011). Da pulverização ao monopólio da violência: expansão e consolidação do Primeiro Comando da Capital (PCC) no sistema carcerário paulista. ( Tesis inédita de doctorado). Universidad de Sao Paulo, Sao Paulo.

Cornblit, O. (2001). Violencia social, genocidio y terrorismo. Buenos Aires: Fondo de Cultura Económica.

Concha, A. (2002). "Salud, Violencia e inseguridad". En Carrión, F., editor, Seguridad Ciudadana, ¿Espejismo o realidad? (pp. 503-520). Quito: FLACSO.

Crettiez, X. (2009). Las formas de la violencia. Buenos Aires: Waldhunter Editores.

Da Silva, L. (2003). Pronunciamento do presidente da República, Luiz Inácio Lula da Silva, na sessão solene de posse no Congresso Nacional, Brasília - DF, Presidencia de la república. Recuperado de: http://www.biblioteca.presidencia.gov.br/ex-presidentes/luiz-inacio-lulada-silva/discursos/1o-mandato/pdfs-2003/1o-semestre/01-01-2003-pronunciamentodo-presidente-da-republica-luiz-inacio-lula-da-silva-na-sessao-solene-de-posse-nocongresso-nacional/view?searchterm $=$ Pronunciamento $\% 20 \mathrm{do} \% 20$ presidente $\% 20 \mathrm{da} \% 20$ República,\%20Luiz\%20Inácio\%20Lula\%20da\%20Silva,\%20na\%20sessão\%20solene\%20 de\%20posse\%20no\%20Congresso\%20Nacional Consultado el 10.9.2014. 
Dammert, L. (2011). La violencia se ha convertido en la forma de resolución de los conflictos. Emol. Recuperado de: http://www.emol.com/tendenciasymujer/Noticias/2011/12/01/22002/LuciaDammert-La-violencia-se-ha-convertido-en-la-forma-de-resolucion-de-los-conflictos.aspx

Dammert, L. \& Zúñiga, L. (2007). Seguridad y violencia: desafíos para la ciudadanía. Santiago de Chile: FLACSO.

Dowdney, L. (2003). Niños en el tráfico de drogas. Un estudio de casos de niños envueltos en la violencia armada organizada en Río de Janeiro. Río de Janeiro:Viva Río.

Elias, N. (2002). Escritos \& ensaios 1. Rio de Janeiro: Jorge Zahar.

Elias, N. \& Scotson, J. (2000). Os estabelecidos e os outsiders. Rio de Janeiro: Jorge Zahar.

Escalante, J. (2003). La delincuencia organizada y su influencia en la seguridad defensa hemisférica. Washington DC: Colegio Interamericano de Defensa.

Exército brasilero (2010). O processo de Transformação do exército. Brasilia: Estado-Maior do Exército.

Fleitas, D. (2014). Delito y Violencia en América Latina y el Caribe. Perfil de los países de la región. Buenos Aires: Asociación de Políticas Públicas.

Galtung, J. (2004). "Violencia, guerra y su impacto. Sobre los efectos visibles e invisibles de la violencia" Polylong Foro para filosofía Intercultural. Recuperado de: http://them.polylog. org/5/fgj-es.htm Consultado el 2.6.2014.

Martinic, R. (2009). Nuevas tendencias en las modalidades de empleo de la fuerza en los países del Cono Sur ampliado: Análisis de datos. Santiago de Chile: Academia de Guerra del Ejército de Chile.

Mazo, D. \& López, J. (2009). "Narcotráfico y paternalismo en Rio de Janeiro" Política y Sociedad 46 (1 y 2$), 205-219$.

Ministerio de Defensa de Brasil (2005). Desafios na Atuação das Forças Armadas. Brasilia: Secretaria de estudios e cooperação.

(2014). Garantia da Lei da Orden. Brasilia: Ministério da Defesa Estado-Maior Conjunto das Forças Armadas.

Morrison, M. Buvinic, A. \& Shifter, M. (2005). "América Violenta: Factores de riesgo, consecuencias e implicaciones para las políticas sobre la violencia social y doméstica". 
En Fruhling, H. Tulchin, J. \&Golding, H. (eds.) Crimen y Violencia en América Latina, ( pp. 117-151). Colombia:Fondo de Cultura Económica.

Koonings, K. \& Veenstra, S. (2007). ). "Exclusión social, actores armados y violencia urbana en Río de Janeiro". En Foro Internacional XLVII (3), 616-636.

Sodré, M. (2001). Sociedad, cultura y violencia. Buenos Aires: Grupo Editorial Norma.

Olavarria, M. (2013). "Costos del Delito y la Violencia: conceptos y métodos". En Revista Estudios de Seguridad y Defensa 1, 43-89.

Reichenheim, M.; Ramos de Souza, E.; Leite, C.; Prado de Mello, M.; Furtado, C. y De Souza, M. (2011). "Health in Brazil 5: Violence and injuries in Brazil: The effect, progress made, and challenges ahead". En The Lancet, 377 (9781), 1962 -1975. Recuperado de: http:// www.thelancet.com/journals/lancet/article/PIIS0140-6736(11)60053-6/fulltext\# article_ upsell Consultado el 10.9.2014.

República Federativa de Brasil (2008). Estrategia Nacional de Defensa. Paz y seguridad para Brasil. Brasilia: Ministerio de Defensa.

(2012). Livro Blanco de Defensa Nacional. Brasilia: Ministerio de

Defensa.

Rousseff, D. (2011, abril 5). Discurso de la Presidenta da República, Dilma Rousseff, durante la cerimonia de presentación de las insígnias de las Órdenes de Mérito de la Defensa de la Fuerzas Armada - Brasília/DF. Recuperado de: http://www2.planalto.gov.br/imprensa/ discursos/discurso-da-presidenta-da-republica-dilma-rousseff-durante-cerimonia-deapresentacao-das-insignias-das-ordens-do-merito-da-defesa-e-das-forcas-armadas Consultado el 22.2.2012.

(2012a, febrero, 01). Discurso de la presidenta Dilma Rousseff en la sesión especial de obertura del año judicial 2012 en el Supremo Tribunal Federal - Brasília/DF. Recuperado de: http://www2.planalto.gov.br/imprensa/discursos/discurso-do-presidente-da-republicaem-exercicio-michel-temer-na-sessao-especial-de-abertura-do-ano-judiciario-de-2012do-supremo-tribunal-federal-stf-brasilia-df Consultado el 19.2.2012.

(2012b). Mensagem ao Congresso Nacional, 2011: 2a Sessão Legislativa Ordinária da 54aa legislatura. Brasília: Presidência da República. Recuperado de: http://www.casacivil. gov.br/noticias/mensagem-ao-congresso-nacional-2012 Consultado el 19.2.2012.

(2013, junio 14). Discurso da Presidenta da República, Dilma Rousseff, durante cerimônia de anúncio de investimentos em infraestrutura urbana e equipamentos sociais nas 
comunidades da Rocinha e nos complexos do Lins e do Jacarezinho, Río de Janeiro. Recuperado de: http://www2.planalto.gov.br/acompanhe-o-planalto/discursos/discursos-da-presidenta/ discurso-da-presidenta-da-republica-dilma-rousseff-durante-cerimonia-de-anuncio-deinvestimentos-em-infraestrutura-urbana-e-equipamentos-sociais-nas-comunidades-darocinha-e-nos-complexos-do-lins-e-do-jacarezinho. Consultado el 12.9.2014.

Troncoso, V. (2014). "El reto del crimen organizado en Brasil y su impacto en las políticas de seguridad y defensa". En Garay, C. Pérez Gil, L. Troncoso, V. (eds.) Gobernabilidad y Seguridad en América Latina: Desafíos del sector Defensa (pp. 185 - 199). Santiago de Chile: Instituto de Estudios Avanzados de la Universidad de Santiago de Chile, Recuperado de: http://seguridadcondemocracia.org/administrador_de_carpetas/biblioteca_virtual/Gobernabilidad_y_Seguridad_Defensa_en_AL.pdf Consultado el 10.8.2014.

UNODC (2014). World Drug Report 2014. Viena: United Nations Publication.

Waiselfisz, J. (2011). Mapa da Violência 2012. Os Novos Padrões da Violência Homicida no Brasil. Sao Paulo: Instituto Sangari. 